\title{
Anterior uveitis associated with Mycoplasma pneumoniae pneumonia
}

\author{
G.M.B. Dawidek
}

Department of Medicine, St Helier Hospital, Carshalton, Surrey SM5 IAA, UK

\begin{abstract}
Summary: A case of Mycoplasma pneumoniae pneumonia complicated by anterior uveitis is described. Anterior uveitis as a complication has not been reported before. Its possible mechanism is discussed.
\end{abstract}

\section{Introduction}

Mycoplasma pneumoniae is a mollicute that can cause atypical pneumonia. This may be accompanied by various extrapulmonary disorders. ${ }^{1}$ Many systems can be affected. Clinical conditions affecting joints, skin, gastrointestinal tract, heart, and haematopoietic and nervous systems have all been described. The patient reported here suffered from anterior uveitis. Search of the literature has revealed no reference to this complication.

\section{Case report}

A 36 year old man was admitted with a 2 week history of a productive cough and intermittent pyrexia, with no history of systemic or ocular disease. On examination he was febrile. There were signs of pneumonia at the right lung base confirmed on X-ray. Cold agglutinins were present at a titre of 1 in 2000. Antinuclear and rheumatoid factors were negative. A diagnosis of $M$. pneumoniae pneumonia was made. Oral tetracycline was prescribed.

His condition improved rapidly but 3 days later he developed a macular erythematous rash distributed bilaterally over the lower legs and soles of the feet. The lesions were flat and non-indurated. Next day he complained of soreness in both eyes and photophobia. Conjunctivitis was diagnosed and topical chloramphenicol prescribed. Three days later there was no improvement and an ophthalmological opinion was sought. Ophthalmological examination confirmed a normal visual acuity.

\footnotetext{
Correspondence: G.M.B. Dawidek, M.R.C.P., Ophthalmology Department, University Hospital of Wales, Heath Park, Cardiff CF4 4XW, UK.

Accepted: 12 November 1990
}

There was bilateral ciliary injection with flare and $2+$ cells in both anterior chambers. Intraocular pressures and fundoscopy were normal. An anterior chamber tap for organisms was not considered clinically desirable. A diagnosis of acute anterior uveitis was made and topical steroids and mydriatics commenced.

Two weeks after admission he was well and was discharged on oral medication for 1 week. The anterior uveitis had subsided. No anterior chamber activity was present and his topical medication was reduced. Serum antimycoplasmal IgG had risen from a titre of 1 in 20 on admission to 1 in 640, and the antimycoplasmal IgM, which had been negative, was positive. Two weeks later the antimycoplasmal IgG had fallen to 1 in 80 .

Four weeks later he was readmitted with fever, an erythematous rash on both arms, similar in morphology to that noted on his first admission, pain in the wrists, and a return of soreness in the eyes and photophobia. Anterior chamber activity was present and the antimycoplasmal IgG had returned to 1 in 640 . A relapse was diagnosed. He was treated with erythromycin and the application of topical steroids to his eyes increased. His symptoms improved rapidly and he was discharged 5 days later.

There was no further recurrence of his systemic symptoms. The uveitis, however, persisted and 3 months after initial presentation, despite continuing topical steroids, signs of anterior chamber activity remained. A month later all activity had subsided and he has remained well since. Tissue typing subsequent to recovery showed him to be HLA B27 negative.

\section{Discussion}

This patient had pneumonia and a high titre of cold agglutinins, and developed a rise in antimycoplas- 
mal IgG and positive antimycoplasmal IgM, all of which were consistent with atypical pneumonia caused by $M$. pneumoniae. However, this infection was complicated by a rash, arthralgia and anterior uveitis, the first two of which are recognized complications. ${ }^{1}$ Uveitis has not been reported previously but its recurrence with the relapse strongly suggests it was associated with $M$. pneumoniae pneumonia in this case.

The possibility that anterior uveitis in this patient was related to an underlying connective tissue disorder or another systemic disease such as sarcoidosis has to be considered. However, he had previously been well, anti-nuclear and rheumatoid factors were negative, and the chest X-ray showed no signs of sarcoidosis. The possibility is therefore considered remote.

A variety of skin rashes including erythema nodosum have been reported in association with $M$. pneumoniae pneumonia. ${ }^{2}$ The erythematous rashes described above were distinct from erythema nodosum in that they were not raised and the first rash involved the soles of the feet. They were considered to be a complication of the mycoplasmal pneumonia, although the possibility that the initial rash was a drug reaction was also considered.

Circulating immune complexes may initiate some cases of uveitis. ${ }^{3}$ A type III hypersensitivity reaction may occur when they are deposited in vessel walls in the ciliary body. Complement is attracted and inflammation results. Circulating immune complexes have been identified in the serum of patients with $M$. pneumoniae pneumonia. ${ }^{4}$ They may have caused the uveitis in this case as well as the rashes and arthralgia.

Alternatively, the anterior uveitis may have been caused by direct invasion of the eye by $M$. pneumoniae. Although anterior chamber tap for organisms was not considered clinically desirable, some evidence exists to support this hypothesis. Rats inoculated with Mycoplasma arthritidis develop an anterior uveitis, conjunctivitis and an arthritis. ${ }^{5}$ The mollicutes have been localized to the stroma of the ciliary body by binding and indirect immunofluorescence of monoclonal antibodies. Furthermore, in a case of $M$. pneumoniae pneumonia associated with neurological complications, $M$. pneumoniae has been identified in cerebrospinal

\section{References}

1. Taylor-Robinson, D. Mycoplasmas. In: Weatherall, D.J., Ledingham, J.G.G. \& Warrell, D.A. (eds). Oxford Textbook of Medicine, 2nd ed. Oxford University Press, Oxford, 1987, pp. $5.366-5.373$

2. Grayston, J.T., Alexander, E.R., Kenny, G.E., Clarke, E.R., Fremont, J.C. \& MacColl, W.A. Mycoplasma pneumoniae infections. JAMA 1965, 191: 369-374. fluid by indirect fluorescent antibody and radioisotope uptake techniques. ${ }^{6}$

Organisms morphologically similar to extracellular mollicutes such as $M$. pneumoniae can be identified within cells. Confirmation by culture is not possible. They are therefore termed mollicutelike organisms (MLO). Recently such MLO have been identified in biopsies from patients with ocular inflammation. In 3 cases of chronic uveitis associated with Crohn's disease vitreous leucocytes demonstrated changes in their ultrastructure characteristic of colonization by MLO. $^{7}$ Similar changes have also been demonstrated in leucocytes from 3 cases of orbital pseudotumour. ${ }^{8}$ Thus, direct invasion of the uveal tract by $M$. pneumoniae may have produced anterior uveitis.

Micro-organisms may have a surface structure close to that of a specific HLA antigen - this is termed 'molecular mimicry'. 'Patients with a particular HLA antigen may mount an immune response to an invading micro-organism that crossreacts with and damages their own tissues. The HLA B27 antigen is present in over $50 \%$ of patients with acute anterior uveitis. ${ }^{10}$ Acute anterior uveitis has been shown to occur in patients who are HLA B27 positive and have suffered Yersinia enterocolitica infection. ${ }^{11}$ It has also been shown to be associated with positive stool cultures for Klebsiella pneumoniae in patients with ankylosing spondylitis, the majority of whom were positive for HLA B27. ${ }^{12}$ Therefore, molecular mimicry may occur between the HLA B27 antigen and a microorganism to produce anterior uveitis. It is unlikely, however, that molecular mimicry could have occurred in the case reported above, as the patient was negative for HLA B27.

This case report has described the recurrent association of anterior uveitis with $M$. pneumoniae pneumonia. The anterior uveitis may have been caused by a type III hypersensitivity reaction to circulating immune complexes or it may have been due to direct invasion of ocular tissues by $M$. pneumoniae.

\section{Acknowledgement}

I thank Dr M.R. Bending, F.R.C.P. for allowing me to report on his patient and for arranging the tissue typing.

3. O'Connor, G.R. Factors related to the initiation and recurrence of uveitis. Am J Ophthalmol 1983, 96: 577-599.

4. Biberfeld, G. \& Norberg, R. Circulating immune complexes in Mycoplasma pneumoniae infection. J Immunol 1974, 112: 413-415. 
5. Thirkill, C.E., Song, D.Y. \& Gregerson, D.S. Application of monoclonal antibodies to detect intraocular mycoplasma antigens in Mycoplasma arthritidis-infected Sprague-Dawley rats. Infect Immun 1983, 40: 389-397.

6. Bayer, A.S., Galpin, J.E., Theofilopoulos, A.N. \& Guze, L.B. Neurologic disease associated with Mycoplasma pneumoniae pneumonitis. Demonstration of viable Mycoplasma pneumoniae in cerebrospinal fluid and blood by radioisotopic and immunofluorescent tissue culture techniques. Ann Intern Med 1981, 94: 15-20.

7. Johnson, L.A., Wirostko, E. \& Wirostko, W.J. Crohn's disease uveitis: parasitisation of vitreous leucocytes by mollicute-like organisms. Am J Clin Pathol 1989, 91: 259-264.

8. Wirostko, E., Johnson, L. \& Wirostko, B. Chronic orbital inflammatory disease: parasitisation of orbital leucocytes by mollicute-like organisms. $\mathrm{Br} J$ Ophthalmol 1989, 73: 865-870.
9. Michalski, J.P. \& Biundo, J.J. Histocompatibility antigens. In: Katz, W.A. (ed.) Diagnosis and Management of Rheumatic Diseases, 2nd ed. J.B. Lippincott, Philadelphia, 1988, p. 242.

10. Brewerton, D.A., Caffrey, M., Nicholls, A., Walters, D. \& James, D.C.O. Acute anterior uveitis and HL-A 27. Lancet 1973, ii: 994-996.

11. Saari, K.M., Laitinen, O., Leirisalo, M. \& Saari, R. Ocular inflammation associated with Yersinia infection. Am J Ophthalmol 1980, 89: 84-95.

12. Ebringer, R., Cawdell, D. \& Ebringer, A. Klebsiella pneumoniae and acute anterior uveitis in ankylosing spondylitis. Br Med J 1979, 1: 383. 